\title{
Anti-cancer vaccine candidates in specific immunotherapy for bladder carcinoma
}

\author{
YOSHIHIRO KOMOHARA ${ }^{1,2}$, MAMORU HARADA $^{1,2}$, YOSHIMI ARIMA $^{1}$, SHIGETAKA SUEKANE $^{3}$, \\ MASANORI NOGUCHI ${ }^{3}$, AKIRA YAMADA $^{1,4}$, KYOGO ITOH $^{2,4}$ and KEI MATSUOKA ${ }^{3}$ \\ ${ }^{1}$ Cancer Vaccine Development Division, Kurume University Research Center for Innovative Cancer Therapy, \\ Departments of ${ }^{2}$ Immunology, ${ }^{3}$ Urology, Kurume University School of Medicine; ${ }^{4}$ Center of the 21 st Century \\ COE Program for Medical Science, Kurume University, Kurume, Fukuoka 830-0011, Japan
}

Received June 20, 2006; Accepted August 7, 2006

\begin{abstract}
We have previously identified numerous tumorrejection antigens and their epitope peptides having the potential to induce cancer-reactive cytotoxic $\mathrm{T}$ lymphocytes (CTLs) in patients with various types of cancer. In the present study, we attempted to determine which antigens and their peptides are useful in specific immunotherapy for bladder carcinoma $(\mathrm{BC})$ patients, especially those with human leukocyte antigen (HLA)-A24+ alleles. The mRNA expression of a panel of cancer-associated antigens was examined regarding four $\mathrm{BC}$ cell lines. As a result, three candidate antigens, including SART3, multidrug resistance-associated protein 3 (MRP3), and polycomb group protein enhancer of zeste homolog 2 (EZH2), were expressed in three of four BC cell lines. Thereafter, antigen-derived peptides which we reported to induce cancer-reactive CTLs from HLA-A $24^{+}$patients with various types of cancer were examined for their potential to induce CTLs from peripheralblood mononuclear cells of HLA-A24+ BC patients. Among these antigen-derived six peptides, SART3 ${ }_{109-118}, \mathrm{MRP}_{1293-1301}$, and $\mathrm{EZH} 2_{735-742}$ peptides efficiently induced peptide-specific and BC cell-reactive CTLs from HLA-A24+ $\mathrm{BC}$ patients. The cytotoxicity against $\mathrm{BC}$ cells was dependent on peptidespecific $\mathrm{CD}^{+} \mathrm{T}$ cells. IgG reactive to the SART3 ${ }_{109-118}$ peptide was frequently detected in the plasma of BC patients. This information could facilitate the development of effective peptide-based immunotherapy for HLA-A24+ BC patients.
\end{abstract}

Correspondence to: Dr Yoshihiro Komohara, Cancer Vaccine Development Division, Kurume University Research Center for Innovative Cancer Therapy, Kurume University School of Medicine, 67 Asahi-machi, Kurume, Fukuoka 830-0011, Japan

E-mail: ycomo@med.kurume-u.ac.jp

Key words: peptide vaccine, HLA-A24, bladder cancer, SART3, MRP3, EZH2

\section{Introduction}

Approximately $75 \%$ of bladder carcinoma (BC) are of a superficial type and can be surgically removed. However, two-thirds of patients will develop tumor recurrence within 5 years (1). After the successful treatment of superficial bladder cancer with Bacillus Calmette-Guerin (BCG) was established in 1976, BCG therapy has been considered to be the most successful protocol to $\mathrm{BC}$ (2). On the other hand, cytokines, including interferons (IFNs), interleukin (IL)-2, and IL-12, were demonstrated as new effective immunotherapeutic agents for superficial BC in several clinical trials (3). In contrast to non-specific immunotherapy, recent advances in tumor-rejection antigens (4), and such antigen-derived peptides, recognized by cancer-reactive cytotoxic $\mathrm{T}$ lymphocytes (CTLs), have been applied in clinical trials of anticancer vaccine (5-8). However, information regarding vaccine candidates applicable to specific immunotherapy to $\mathrm{BC}$ is very limited.

We identified a panel of tumor antigens and their epitope peptides that are currently known to be recognized by cancer-reactive CTLs. One representative is SART3, and SART3-derived peptides possess the potential to induce human leukocyte antigen (HLA) class I-restricted CTLs in patients with several types of epithelial cancer (9-12). We have also reported that antigenic peptides derived from multidrug resistance-associated protein 3 (MRP3) and polycomb group protein enhancer of zeste homolog 2 (EZH2) can induce HLA class I-restricted CTLs from HLA-A24+ patients with various types of cancer $(13,14)$. In the present study, we attempted to determine which antigens and their peptides are useful in specific immunotherapy for HLA-A24+ BC patients among a panel of tumor antigens and their peptides identified thus far.

\section{Materials and methods}

Patients. The study protocol (Protocol \# 2484) was approved by the Institutional Ethics Review Board of Kurume University, and informed written consent was obtained from all the subjects from whom peripheral blood mononuclear cells (PBMCs) were obtained in this study. PBMCs 
were prepared by Ficoll-Conray density gradient centrifugation and cryopreserved until they were used for the experiments. The expression of HLA-A24 molecule on PBMCs of cancer patients was determined by flow cytometry as described previously (15).

Cell lines. The bladder cell carcinoma cell lines, HT1376, EJ-1, UM-UC-3, and Tcc-SUP, were incubated in RPMI-1640 supplemented $10 \%$ fetal bovine serum (FBS). C1R-A24 is an HLA-A*2402-expressing C1R cell line (Dr M. Takiguchi, Kumamoto University, Japan). The HLA-A*2402-transfected cell line, HT1376-A24, was established as described previously (16).

Reverse transcriptase-polymerase chain reaction (RT-PCR). Total RNA was isolated from cancer cell lines using RNAzol $^{\mathrm{TM}} \mathrm{B}$ (Tel-Test Inc., Friendswood, TX). The cDNA was prepared using the SuperScript ${ }^{\mathrm{TM}}$ Preamplification System for first-strand cDNA synthesis (Invitrogen, CA), and was amplified using the following primers: 5'-AAGT ACGCCAACATGTGGC-3' (sense) and 5'-CTCTGCTCA TTGACACGAGC-3' (anti-sense) for SART3, 5'-TGCCTG CTTCAAGCTTATCC-3' (sense) and 5'-GGTGATAACCA GAGCCTTCC-3' (anti-sense) for MRP3, 5'-GCCATTTCCT CAATGTTTCC-3' (sense) and 5'-TGGTCCACAAGGTT TGTTGT-3' (anti-sense) for EZH2, 5'-ATAGACACCAACC GCTCTCG-3' (sense) and 5'-GGTCCACCACTGACA CGTTG-3' (anti-sense) for GAPDH. RT-PCR amplification of SART1, SART2, Her2/neu, CypB, ART1, ART4, CEA, and EGFR were done as described previously (17-20).

Induction of peptide-specific CTLs from PBMCs. Peptides with $>90 \%$ purity were purchased from Biologica Co. (Nagoya, Japan). Influenza (Flu) virus, EB virus (EB), and HIV-derived peptides were used as controls binding to HLA-A24 alleles, as described previously (9). Assays for the detection of peptide-specific CTLs were performed according to a previously reported method with several modifications (21). Briefly, PBMCs were incubated with $10 \mu \mathrm{g} / \mathrm{ml}$ of each peptide in quadruplicate in a 96-well microplate (Nunc, Roskilde, Denmark). The culture medium consisted of 45\% RPMI-1640, 45\% AIM-V medium (Life Technologies, Gaithersburg, MD), 10\% FBS, 100 units $/ \mathrm{ml}$ IL-2, and 0.1 mM MEM non-essential amino acid solution (Life Technologies). On the 15th day of culture, cells were separated into 4 wells, and then cultured with the corresponding peptide-pulsed C1R-A24 cells. The HIV peptide was used a negative control. After an 18-h incubation, the IFN- $\gamma$ production was determined by ELISA.

Cytotoxicity assay. Peptide-stimulated PBMCs were tested for their cytotoxicity against BC cell lines, HT1376 (HLAA24 negative) and HT1376-A24 (HLA-A24 positive), by a standard 6-h ${ }^{51} \mathrm{Cr}$-release assay. Phytohemagglutinin (PHA)activated $\mathrm{T}$ cells from HLA-A24 positive healthy donors were used as a negative control. After $\mathrm{CD} 8^{+} \mathrm{T}$ cells were positively isolated using a CD8-positive isolation kit (Dynal, Oslo, Norway), two-thousand ${ }^{51} \mathrm{Cr}$-labeled cells per well were cultured with effector cells in 96-round-well plates. The specific ${ }^{51} \mathrm{Cr}$-release was calculated, as described
Table I. Results of RT-PCR analysis of cancer-associated antigens expressed in $\mathrm{BC}$ cell lines.

\section{EJ-1 UM-UC-3 HT1376 Tcc-SUP PBMC}

\begin{tabular}{llllll}
\hline SART1 & + & + & + & + & + \\
SART2 & + & + & + & + & + \\
SART3 & + & + & + & \pm & - \\
MRP3 & + & + & + & - & - \\
EZH2 & + & + & + & \pm & - \\
Her2/neu & + & + & + & - & - \\
CypB & + & + & + & + & + \\
ART-1 & + & + & + & - & \pm \\
ART-4 & + & + & + & \pm & \pm \\
CEA & + & + & + & \pm & - \\
EGFR & \pm & \pm & + & - & - \\
\hline
\end{tabular}

Intensity of RT-PCR products was judged as follows: -, not detectable; \pm , weakly detectable; + , strongly detectable. SART, squamous-cell carcinoma antigen recognized by $\mathrm{T}$ cell; CypB, cyclophilin B; ART, ADP-rebosyltransferase; CEA, carcinoembryonic antigen; EGFR, epidermal growth factor receptor; PBMC, peripheral blood mononuclear cell.

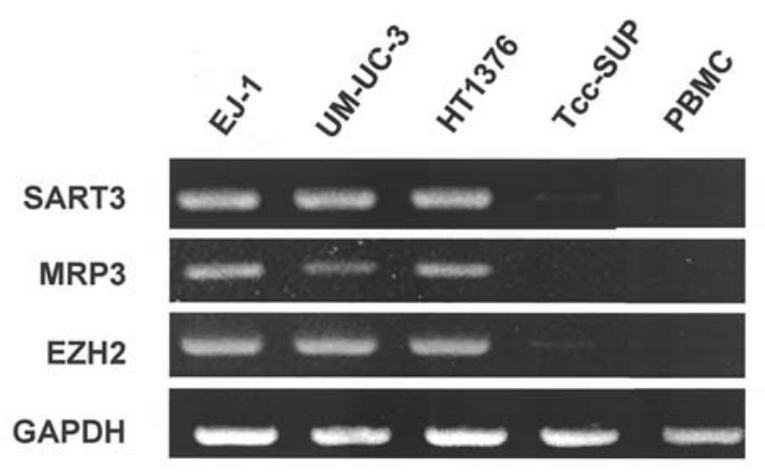

Figure 1. mRNA expression of cancer-associated antigens in BC cell lines. RT-PCR was performed using cDNA from four RCC cell lines, EJ-1, UMUC-3, HT1376, and Tcc-SUP. GAPDH was used as an internal control.

previously (22). The specificity of peptide-stimulated PBMCs was confirmed by a cold inhibition assay. Twenty-thousand unlabeled C1R-A24 cells, which were pre-pulsed with either the HIV peptide or a corresponding peptide, were used as cold target cells.

Detection of peptide-specific immunoglobulin G. Peptidespecific immunoglobulin $\mathrm{G}(\mathrm{IgG})$ levels in plasma were measured by ELISA as previously reported (22).

\section{Results}

mRNA expression of three cancer-associated antigens in $B C$ cell lines. To date, we have identified a panel of cancerassociated antigen and their epitope peptides. These antigens include SART1 SART2, SART3, MRP3, EZH2, ART1, 

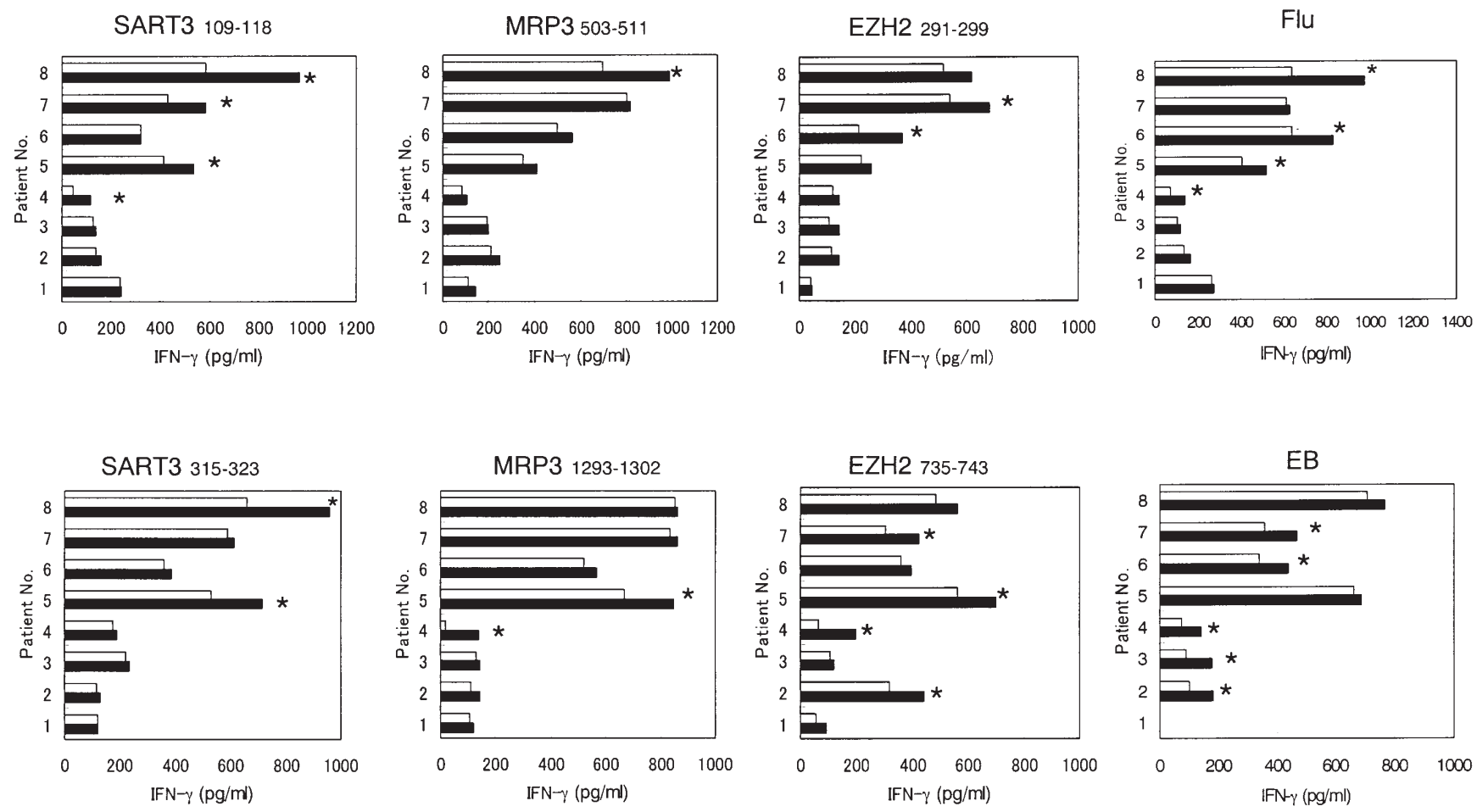

Figure 2. Induction of peptide-reactive CTLs from the PBMCs of HLA-A24+ BC patients. The PBMCs that were stimulated in vitro with each of the indicated peptides were tested for their reaction to C1R-A24 cells which were pre-pulsed with the indicated peptide (closed) or control HIV peptide (open). The assay was performed in duplicate. $\mathrm{P}<0.05$ statistically significant (Student's t-test).

ART4, Her2/neu, CypB, CEA, and EGFR (13-20,22-26). We first performed RT-PCR analysis on four BC cell lines, HT1376, EJ-1, UM-UC-3, and Tcc-SUP, and normal PBMCs. These cancer-associated antigens were expressed in most of the four BC cell lines, and results are summarized in Table I. Because mRNA expression of SART1, SART2, ART1, ART4, and $\mathrm{CypB}$ were detected in normal PBMCs, and because Her2/neu, CEA, and EGFR are well characterized antigens by other investigators (27-29), we focused on three antigens SART3, MRP3, and EZH2, in the following experiments. The results of RT-PCR on these three antigens are shown in Fig. 1.

Induction of peptide-specific CTLs from $H L A-A 24^{+} B C$ patients. We next attempted to determine whether or not these antigen-derived peptides could have the potential to generate peptide-specific CTLs from the PBMCs of HLA-A24+ BC patients. Because we have already identified their epitope peptides possessing the ability to induce cancer-reactive CTLs from HLA-A $24^{+}$patients with other types of cancer (12-14), we utilized the following six peptides: SART3 ${ }_{109-118}$, $\mathrm{SART}_{315-323}, \mathrm{MRP}_{503-511}, \mathrm{MRP} 3_{1293-1301}, \mathrm{EZH} 2_{291-299}$, and EZH $2_{735-742}$. The PBMCs from HLA-A24+ $\mathrm{BC}$ patients were stimulated in vitro with each of the peptides or control peptides, and were then examined for IFN- $\gamma$ production in response to the corresponding peptide-pulsed C1R-A24 cells. The result was that these six peptides induced corresponding peptide-reactive CTLs in $4,2,1,2,2$, and 4 of 8 BC patients (Fig. 2). Regarding each cancer-associated antigen, the SART3 ${ }_{109-118}, \mathrm{MRP}_{1293-1301}$, and EZH2 ${ }_{735-742}$ peptides appeared to be superior to another counterpart peptide. Flu and EBV peptides were used as positive controls. The induction rate of the SART3 ${ }_{109-118}$ and EZH2 ${ }_{735-742}$ peptides was comparable to that of the control peptides. In total, three peptide candidates, the SART3 $3_{109-118}, \mathrm{MRP}_{1293-1301}$, and $\mathrm{EZH} 2_{735-742}$, were further examined in the flowing experiments.

Cytotoxicity of peptide-stimulated PBMCs against BC cells. We next determined whether or not CTLs induced by in vitro stimulation with peptides could exhibit cytotoxicity against BC cells. The PBMCs from patients 5 and 8 were stimulated in vitro with each of the three peptides, SART3 $3_{109-118}$, MRP3 ${ }_{1293-130}$, and EZH2 $2_{735-742}$, and their cytotoxicity against HLA-A24- HT1376 cells, and its HLA-A24-expressing transfectant cells, HT1376-A24, was measured. The PHAstimulated $\mathrm{T}$ cell blasts from HLA-A24+ healthy donors were used as a control for HLA-A24-expressing and antigennegative cells. As shown in Fig. 3A, the PBMCs from patient 5 that were stimulated in vitro with either the MRP $3_{1293-1302}$ or EZH2 ${ }_{735-743}$ peptide exhibited a higher level of cytotoxicity against HT1376-A24 cells than against HT1376 cells and HLA-A24 ${ }^{+} \mathrm{T}$ cell blasts. The PBMCs from patient 8 that were stimulated in vitro with the SART3 $3_{109-118}$ peptide also showed a higher level of cytotoxicity against HT1376-A24 cells than against HT1376 cells and HLA-A24+ $\mathrm{T}$ cell blasts. These results indicate that CTLs that were stimulated in vitro with each of the three peptides could exhibit cytotoxicity against BC cells in an HLA-A24-restricted manner.

Peptide-specific and $C D 8^{+} T$ cell-dependent cytotoxicity against $B C$ cells. We further tried to identify cells responsible for the 
A

Pt. 8 SART3 109-118

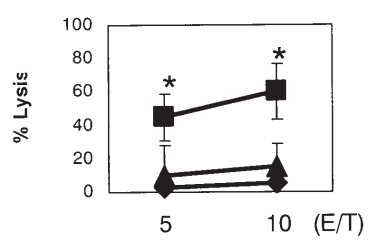

Pt. 5 MRP3 1293-1302

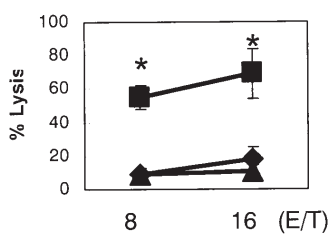

Pt. 5 EZH2 $735-743$

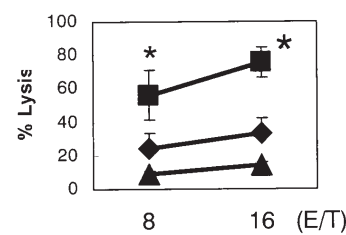

HT1376 - HT1376-A24 \ PHA Blast

\section{B}

Pt. 8 SART3 109-118

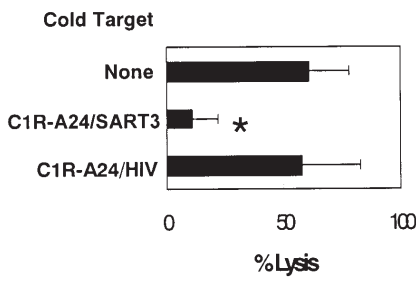

Pt. 5 MRP3 1293-1302

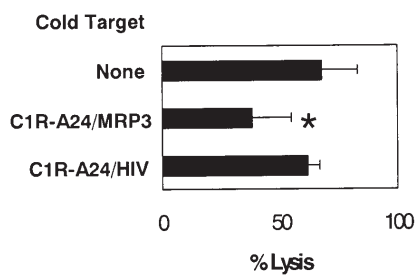

Pt. 5 EZH2 735-743

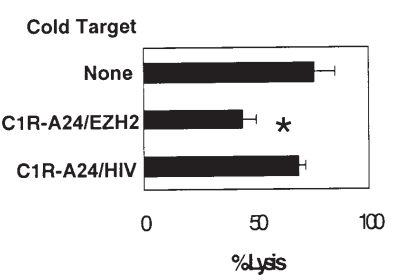

Figure 3. Cytotoxicity of peptide-stimulated PBMCs from HLA-A24+ BC patients was dependent on peptide-specific CD8 ${ }^{+} \mathrm{T}$ cells. (A) Peptide-stimulated PBMCs from patients 5 and 8 were tested for their cytotoxicity toward two BC cell lines (HLA-A24+ HT1376-A24 and HLA-A24- HT1376), and phytohemagglutinin (PHA)-stimulated HLA-A24+ $\mathrm{T}$ cell blasts by $6-\mathrm{h}{ }^{51} \mathrm{Cr}$-release assay. (B) $\mathrm{CD}^{+} \mathrm{T}$ cells from peptide-stimulated PBMCs of HLA-A24+ $\mathrm{BC}$ patients were tested for cytotoxicity against HT1376-A24 cells in the presence of unlabeled C1R-A24 cells, which were preloaded with either the corresponding peptide or the HIV peptide. ${ }^{*} \mathrm{P}<0.05$ statistically significant (Student's t-test).

cytotoxicity of peptide-stimulated CTLs. The cytotoxicity against HLA-A24+ and corresponding antigen-expressing HT1376-A24 cells was significantly suppressed by the addition of corresponding peptide-pulsed unlabeled C1RA24 cells, but not by control HIV peptide-pulsed unlabeled C1R-A24 cells (Fig. 3B). These results indicate that the cytotoxicity of peptide-induced CTLs against HLA-A24+ BC cells is dependent on peptide-specific $\mathrm{CD} 8^{+} \mathrm{T}$ cells.

Detection of $\mathrm{IgG}$ reactive to SART3, MRP3, and EZH2derived peptides. We have previously reported that IgGs reactive to CTL-directed peptides are detected in patients with epithelial cancers $(13,14)$, and that increases in the level of peptide-specific $\operatorname{IgG}$ after peptide vaccination are positively correlated with the clinical response and survival of vaccinated patients $(4,23)$. We therefore tested whether IgGs reactive to the SART3-, MRP3-, and EZH2-derived peptides could be detected in the plasma of cancer patients $(n=15)$ and healthy donors $(n=14)$. In BC patients, IgGs reacting to the SART3 $3_{109-118}$ and SART3 $3_{315-323}$ peptides were detected in ten and six of 15 BC patients, respectively (Fig. 4). IgG reactive to either $\mathrm{MRP}_{503-511}$ or $\mathrm{EZH} 2_{735-743}$ peptide was not detected in any of these patients, and IgGs reactive to the $\mathrm{MRP}_{1293-1302}$ and EZH2 ${ }_{291-299}$ peptides were detected in only one and three patients (data not shown). No IgG was detected in any healthy donors (data not shown). These results indicate that the SART3 ${ }_{109-118}$ peptide is efficiently recognized by both the cellular and humoral immune systems.

\section{Discussion}

In our previous study, we identified SART3-, MRP3-, and EZH2-derived peptides that possess the potential to induce
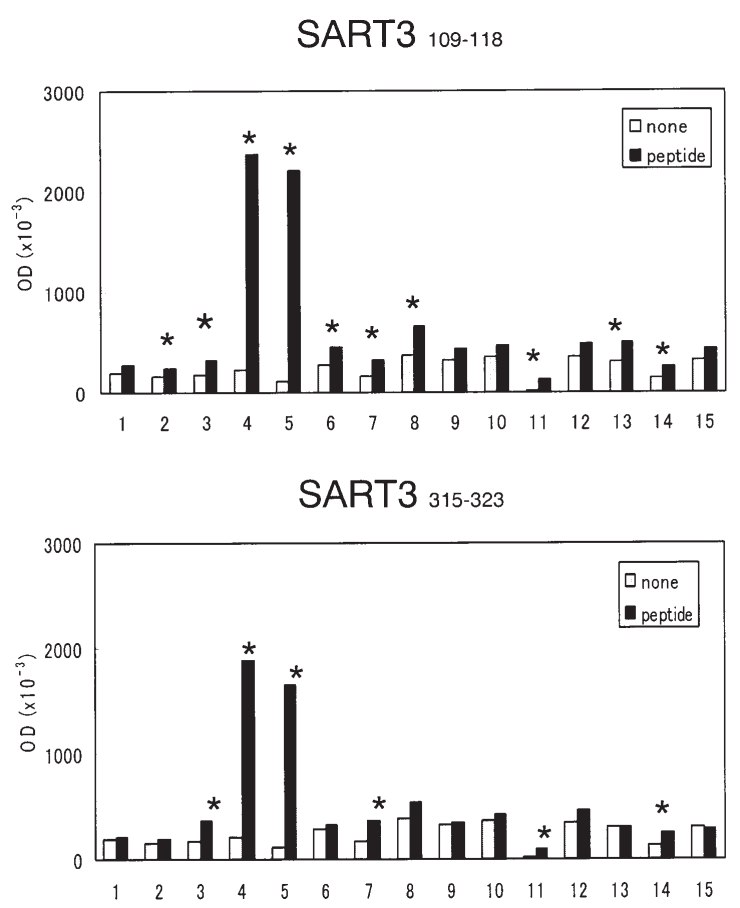

Figure 4. IgGs reactive to the SART3 peptides in the plasma of BC patients. IgG reactive to the corresponding SART3 peptide was judged to be significant when the absorbance in a 1:100-diluted plasma was $>1.5$ times compared to that of no peptide controls. The assay was performed in duplicate and significance was evaluated for each plasma sample. ${ }^{*} \mathrm{P}<0.05$ statistically significant (Student's t-test).

cancer-reactive CTLs from HLA-A24+ patients with various types of cancer other than BC (9-14). As for these cancerassociated antigens, SART3 is expressed in the nucleus of all the malignant tumor cell lines of various histological 
types, whereas no analysis on BC has been done (15). In contrast, MRP3 and EZH2 were reported to be expressed in BC tissues (30-33). MRP3 is a widely-expressed cancerassociated antigen that we identified using tumor-infiltrating lymphocytes into lung adenocarcinoma (14). This antigen seems to be a unique target molecule for cancer vaccines because the expression of MRP3 is associated with multidrug resistance (34), which is an important problem in chemotherapy. On the other hand, EZH2 is a polycomb group protein homologue to the Drosophila enhancer of zeste (35). EZH2 is involved in gene silencing, and dysregulation of this gene-silencing machinery can lead to cancer (36).

In this study, we confirmed the expression of MRP3 and $\mathrm{EZH} 2$ in $\mathrm{BC}$ cell lines, and newly found that three of four bladder carcinoma cell lines were positive for SART3. Furthermore, we showed that these antigen-derived three peptides efficiently induced BC-reactive CTLs from the PBMCs of HLA-A24+ BC patients. A cold inhibition assay clearly revealed that the cytotoxicity toward HLA-A24+ $\mathrm{BC}$ cells was ascribed to peptide-specific $\mathrm{CD}^{+} \mathrm{T}$ cells. Both the preferential expression in $\mathrm{BC}$ cells and the potential of their peptide candidates to induce BC-reactive CTLs suggest that the identified three peptides would be appropriate vaccine candidates for use in specific immunotherapy for HLA-A $24^{+}$BC patients.

We determined whether $\mathrm{IgG}$ reactive to 6 peptide candidates could be detected in $15 \mathrm{BC}$ patients. As a result, IgGs reactive to the SART3 $3_{109-118}$ peptide was detected more frequently compared to the other five peptides. Interestingly, IgGs reactive to the $\mathrm{SART}_{109-118}$ and $\mathrm{SART}_{315-322}$ peptides were detected more frequently in HLA-A24+ $\mathrm{BC}$ patients (Fig. 4, No. 1-8) than in HLA-A24- BC patients (Fig. 4, No. 9-15). In contrast, we previously observed that humoral responses to CTL-directed peptides were not restricted to HLA class I molecules $(37,38)$. These observations seem to be discrepant. Although one explanation is that peptides used for assays were different, we have no clear explanation for this observation at present. We are trying to elucidate roles of peptide-specific IgG in anti-tumor immune responses in cancer patients.

\section{Acknowledgements}

This study was supported in part by Grants-in-Aid from the Ministry of Education, Science, Sports and Culture of Japan (KAKENHI; no. 16591628 to K.M, no. 16790933 to S.S, no 18591449 to M.H, and no. 12213134 to K.I), and from the Ministry of Health, Labor and Welfare, Japan (no. H14trans-002, 11-16, and H12-cancer-004 to K.I, and no. 15-17 to M.H.).

\section{References}

1. Lamm DL: Long-term results of intravesical therapy for superficial bladder cancer. Urol Clin North Am 19: 573-580, 1992.

2. Sohle A and Brandau S: Immune mechanisms in Bacillus Calmette-Guerin immunotherapy for superficial bladder cancer. J Urol 170: 964-969, 2003.

3. Perabo FGE and Muller SC: Current and new strategies in immunotherapy for superficial bladder cancer. Urology 64: 409-421, 2004.
4. Mine T, Sato Y, Noguchi M, et al: Humoral responses to peptides correlate with overall survival in advanced cancer patients vaccinated with peptides based on pre-existing, peptidespecific cellular responses. Clin Cancer Res 10: 929-937, 2004.

5. Vieweg J and Dannull J: Technology insight:vaccine therapy for prostate cancer. Nat Clin Pract Urol 2: 44-51, 2005.

6. Srivastava PK: Therapeutic cancer vaccines. Curr Opin Immunol 18: 201-205, 2006.

7. Lewis JJ: Therapeutic cancer vaccines: using unique antigens. Proc Natl Acad Sci USA 101: 14653-14656, 2004.

8. Pardoll DM: Cancer vaccines. Immunol Today 14: 310-316, 1994.

9. Murayama K, Kobayashi T, Imaizumi T, et al: Expression of the SART3 tumor-rejection antigen in brain tumors and induction of cytotoxic T lymphocytes by its peptides. J Immunother 23: 511-518, 2000.

10. Miyagi Y, Imai M, Sasatomi T, et al: Induction of cellular immune responses to tumor cells and peptides in colorectal cancer patients by vaccination with SART3 peptides. Clin Cancer Res 7: 3950-3962, 2001.

11. Suzuki N, Maeda Y, Tanaka S, Hida N, Mine T, Yamamoto K, Oka M and Itoh K: Detection of peptide-specific cytotixic Tlymphocyte precursors used for specific immunotherapy of pancreatic cancer. Int J Cancer 98: 45-50, 2002

12. Sasatomi T, Suefuji Y, Matsunaga K, et al: Expression of tumor rejection antigens in colorectal carcinomas. Cancer 94: 1636-1641, 2002.

13. Yamada A, Kawano K, Koga M, Matsumoto T and Itoh K: Multidrug resistance-associated protein 3 is a tumor rejection antigen recognized by HLA-A2402-restricted cytotoxic T lymphocytes. Cancer Res 61: 6459-6466, 2001.

14. Ogata R, Matsueda S, Yao A, Noguchi M, Itoh K and Harada M: Identification of polycomb group protein enhancer of zeste homolog 2 (EZH2)-derived peptides immunogenic in HLA-A24 ${ }^{+}$prostate cancer patients. Prostate 60: 273-281, 2004.

15. Kawagoe N, Shintaku I, Yutani S, Etoh H, Matuoka K, Noda S and Itoh K: Expression of the SART3 tumor rejection antigen in renal cell carcinoma. J Urol 164: 2090-2095, 2000.

16. Yang D, Nakao M, Shichijo S, et al: Identification of a gene coding for a protein possessing shared tumor epitopes capable of inducing HLA-A24-restricted cytotoxic T lymphocytes in cancer patients. Cancer Res 59: 4056-4063, 1999.

17. Tanaka S, Tsuda N, Kawano K, et al: Expression of tumorrejection antigens in gynecologic cancers. Jpn J Cancer Res 91: 1177-1184, 2000.

18. Gohara R, Imai N, Rikimaru T, et al: Phase I clinical study of cyclophilin B peptide vaccine for patients with lung cancer. J Immunother 25: 439-444, 2002.

19. Shomura H, Shichijo S, Komatsu N, et al: Identification of epidermal growth factor receptor-derived peptides recognized by both cellular and humoral immune responses in HLA-A24+ non-small cell lung cancer patients. Eur J Cancer 40: 1776-1786, 2004.

20. Ishihara Y, Harada M, Azuma K, et al: HER2/neu-derived peptides recognized by both cellular and humoral immune systems in HLA-A2 ${ }^{+}$cancer patients. Int J Oncol 24: 967-975, 2004.

21. Hida N, Maeda Y, Katagiri K, Takasu H, Harada M and Itoh K: A simple culture protocol to detect peptide-specific T lymphocyte precursors in the circulation. Cancer Immunol Immunother 51: 219-228, 2002.

22. Matsueda S, Takedatsu H, Yao A, Tanaka M, Noguchi M, Itoh $\mathrm{K}$ and Harada $\mathrm{M}$ : Identification of peptide vaccine candidates for prostate cancer patients with HLA-A3 supertype alleles. Clin Cancer Res 11: 6933-6943, 2005.

23. Sato Y, Maeda Y, Shomura H, et al: A phase I trial of cytotoxic T-lymphocyte precursor-oriented peptide vaccines for colorectal carcinoma patients. Br J Cancer 90: 1334-1342, 2004.

24. Shomura H, Shichijo S, Matsueda S, Kawakami T, Sato Y, Todo $\mathrm{S}$ and Itoh $\mathrm{K}$ : Identification of epidermal growth factor receptor-derived peptides immunogenetic for HLA-A2(+) cancer patients. Br J Cancer 90: 1563-1571, 2004.

25. Yamamoto $\mathrm{K}$, Mine $\mathrm{T}$, Katagiri $\mathrm{K}$, et al: Immunological evaluation of personalized peptide vaccination for patients with pancreatic cancer. Oncol Rep 13: 874-883, 2005.

26. Yajima N, Yamanaka R, Mine T, et al: Immunologic evaluation of personalized peptide vaccination for patients with advanced malignant glioma. Clin Cancer Res 11: 5900-5911, 2005. 
27. Memon AA, Sorensen BS, Meldgaard P, Fokdal L, Thykjaer T, and Nexo F: The relation between survival and expression of HER1 and HER2 depends on the expression of HER3 and HER4: a study in bladder cancer patients. Br J Cancer 94: 1703-1709, 2006.

28. La Rue H, Parent-Vaugeois C, Bergeron A, Champetier S and Fradet $\mathrm{Y}$ : Influence of spatial configuration on the expression of carcinoembryonic antigen and mucin antigens in human bladder cancer. Int J Cancer 71: 986-992, 1997.

29. Modjtahedi H, Moscatello DK, Box G, et al: Targeting of cells expressing wild-type EGFR and type-III mutant EGFR (EGFRvIII) by anti-EGFR MAb ICR62: a two-pronged attack for tumor therapy. Int J Cancer 105: 273-280, 2003.

30. Tada Y, Wada M, Migita T, et al: Increased expression of multidrug resistance-associated proteins in bladder cancer during clinical course and drug resistance to doxorubicin. Int J Cancer 98: 630-635, 2002.

31. Raman JD, Mongan NP, Tickoo SK, Boorjian SA, Scherr DS and Gudas LJ: Increased expression of the polycomb group gene, EZH2, in transitional cell carcinoma of the bladder. Clin Cancer Res 11: 8570-8576, 2005.

32. Arisan S, Buyultuncer ED, Palavan-Unsal N, Caskurlu T, Cakir OO and Ergenekon F: Increased expression of EZH2, a polycomb group protein, in bladder carcinoma. Urol Int 75: 252-257, 2005
33. Weikert S, Christoph F, Kollermann J, Muller M, Schrader M, Miller K and Kause H: Expression levels of the EZH2 polycomb transcriptional repressor correlate with aggressiveness and invasive potential of bladder carcinomas. Int J Mol Med 16: 349-353, 2005.

34. Kool M, De Haas M, Scheffer GL, et al: Analysis of expression of cMOAT (MRP2), MRP3, MRP4, and MRP5, homologues of the multidrug resistance-associated protein gene (MRP1), in human cancer cell lines. Cancer Res 57: 3537-3547, 1997.

35. Laible G, Wolf A, Dorn R, et al: Mammalian homologues of the Polycomb-group gene enhancer of zeste mediate gene silencing in Drosophila heterochromatin and at S. cerevisiae telomeres. EMBO J 16: 3219-3232, 1997.

36. Kleer CG, Cao Q, Varambally S, et al: EZH2 is a marker of aggressive breast cancer and promotes neoplastic transformation of breast epithelial cells. Proc Natl Acad Sci USA 100: 11606-11611,2003.

37. Ohkouchi S, Kawamoto N, Koga M, et al: Identification of a CTL-directed epitope encoded by an intron of the putative tumor suppressor gene Testin of the common fragile site $7 \mathrm{G}$ region: a peptide vaccine candidate for HLA-B52 ${ }^{+}$and HLA- $62^{+}$ cancer patients. Eur J Immunol 33: 2964-2973, 2003.

38. Kawamoto N, Yamada A, Ohkouchi S, et al: $\mathrm{IgG}$ reactive to CTL-directed epitopes of self-antigens is either lacking or unbalanced in atopic dermatitis patients. Tissue Antigens 61: 352-361, 2003. 